\title{
A Rapid and Sensitive UHPLC-MS/MS Method for Determination of 2, 3, 8-Trimethylellagic, a Potent Active Compound from Sanguisorba officinalis L., and Its Application in the Pharmacokinetic Study within Thrombocytopenia Rats
}

\author{
Yuqing Wang, ${ }^{1}$ Jianming Wu ${ }^{D},{ }^{1}$ Yunxia Li, ${ }^{2}$ Jing Yang, ${ }^{1}$ Long Wang, ${ }^{1}$ Anguo Wu, \\ Feihong Huang, ${ }^{1}$ Nan Jiang, ${ }^{1}$ Can Tang $\mathbb{D},{ }^{1}$ and Yan Li $\mathbb{C}^{2,3}$ \\ ${ }^{1}$ School of Pharmacy, Southwest Medical University, Luzhou, Sichuan, China \\ ${ }^{2}$ School of Pharmacy, Chengdu University of Traditional Chinese Medicine, Chengdu, China \\ ${ }^{3}$ Institute of Cardiovascular Research, The Key Laboratory of Medical Electrophysiology, Ministry of Education of China, \\ Medical Key Laboratory for Drug Discovery and Druggability Evaluation of Sichuan Province, \\ Luzhou Key Laboratory of Activity Screening and Druggability Evaluation for Chinese Materia Medica, Luzhou, China \\ Correspondence should be addressed to Can Tang; 1984088780@qq.com and Yan Li; 993603736@qq.com
}

Received 13 September 2021; Accepted 16 November 2021; Published 16 December 2021

Academic Editor: Vinod Kumar Tiwari

Copyright (C) 2021 Yuqing Wang et al. This is an open access article distributed under the Creative Commons Attribution License, which permits unrestricted use, distribution, and reproduction in any medium, provided the original work is properly cited.

To investigate the pharmacokinetics of 2, 3, 8-trimethylellagic (TMEA) in rats in vivo and determine the possible effects of the pathological conditions and compatibility, a rapid and sensitive ultra-high-performance liquid chromatography-tandem mass spectrometry (UHPLC-MS/MS) method for quantitative determination was developed. TMEA and Artemetin (internal standard, IS) were separated on an Acquity Shim-pack GIST column with a total running time of 7 min using gradient elution at a flow rate of $0.3 \mathrm{~mL} / \mathrm{min}$. The intraday and interday relative standard deviations were $<9.50 \%$, and the relative error of accuracy was between $-5.70 \%$ and $2.96 \%$. The calibration curve of TMEA demonstrated good linearity with $r^{2}=0.9996$, with the average recovery changing from $94.77 \%$ to $102.47 \%$ and the matrix effect from $93.16 \%$ to $100.15 \%$. Compared with the normal group, the area under the plasma concentration-time curve from time 0 to the last time of quantifiable concentration $\left(\mathrm{AUC}_{(0-t)}\right)$, area under the plasma concentration-time curve from time 0 extrapolated to infinite time $\left(\mathrm{AUC}_{(0-\infty)}\right)$, and the maximum concentration $\left(C_{\max }\right)$ of TMEA increased, whereas the time of maximum concentration $\left(T_{\max }\right)$ and apparent clearance $(\mathrm{CL} / \mathrm{F})$ remarkably decreased in the TMEA group. With significantly reduced $\mathrm{CL} / \mathrm{F}, \mathrm{AUC}_{(0-t)}, \mathrm{AUC}_{(0-\infty)}$, and $C_{\max }$ for TMEA were increased approximately one time after combining with 3, 7-Di-O-methylducheside A (DOMA). $\mathrm{AUC}_{(0-t)}$ and $C_{\max }$ for TMEA in the 2, 3, 8-trimethylellagic-3, 8-dimethoxyellagic acid-2-oxyglucoside (TMEA-DMAG) group were significantly lower than that in the TMEA group with clearly prolonged $T_{\max }$ and increased CL/F. These findings indicate that the changes in the pharmacokinetic parameters of TMEA may be caused by pathological and combination conditions.

\section{Introduction}

Cancer is a disease associated with high global morbidity and mortality rates. It is caused by the abnormal proliferation of cells, a process that is induced by a variety of tumorigenic factors. At present, radiotherapy and chemotherapy are the primary treatment modalities used for malignant tumors. However, as they result in the untargeted killing of cells, such treatments often cause a series of adverse reactions, including nausea, vomiting, diarrhea, skin inflammation, fever, and bone marrow and immunological suppression [1]. In particular, damage to the bone marrow and the suppression of immunologic function lead to the reduction of the leukocyte, erythrocyte, and platelet counts, as well as an increased risk of infection and bleeding. Thrombocytopenia is defined as a condition where the 
platelet count is less than $100 \times 10^{9} / \mathrm{L}$, and its clinical manifestations include splenomegaly, ecchymosis, purpura, superficial lymphadenopathy, intracranial hemorrhage, and death [2]. In addition to radiotherapy and chemotherapy, other processes that contribute to thrombocytopenia include the metastatic cancers of the bone marrow, leukemia, myelodysplastic syndrome, decreased thrombocytopoiesis caused by lesions in the hematopoietic cells, the inactivation of thrombocytopoiesis caused by the deficiency of vitamin B or folate, and increased platelet destruction caused by thrombotic thrombocytopenic purpura [3]. The intravenous infusions of the platelets, hormones, cytokines, and immunoglobulins are common treatments for thrombocytopenia [4]. Despite being temporarily palliative, these treatments are expensive and can lead to serious side effects. Therefore, there is an urgent need to develop safer and more effective drugs for treating thrombocytopenia and reducing the adverse effects of radiotherapy and chemotherapy during the treatment of cancer.

Traditional Chinese medicine is a natural repository from which new active compounds are often discovered. Although there exist several herbal formulations used to treat thrombocytopenia induced by radiotherapy, chemotherapy, or immune thrombocytopenia, only a few reports exist on single herbs and natural medications, such as Panax ginseng C. A. Mey., Ligustrum lucidum Ait., Lycium barbarum L., Spatholobus suberectus Dunn, Agrimonia pilosa Ledeb., Eclipta prostrata L., Sarcandra glabra (Thunb.) Nakai, Dioscorea esculenta (Lour.) Burkill, and Carica papaya L. [5]. Related studies have reported that tannins in Sanguisorba officinalis L. can increase the DNA content and combat myelosuppression in mice with cyclophosphamideinduced thrombocytopenia [6], and the saponins of the herb can enhance the levels of the bone marrow nucleated cells and peripheral blood cells [7]. The mechanism through which these substances promote thrombocytopoiesis is related to the thrombopoietin/thrombopoietin receptor (TPO/ $\mathrm{c}-\mathrm{Mpl}$ ) and the stem cell factor/kit ligand (SCF/C-KIT) pathways. The binding ability of cytokines with their corresponding receptors is thought to be enhanced by the upregulation of TPOR and C-KIT, although it is also possible for pleiotropic cytokines to directly activate TPOR or C-KIT, as well as TPO or SCF [7]. The downstream signal transduction pathway is subsequently activated to promote the megakaryocyte proliferation and differentiation, and the platelet production is further promoted by a series of reactions. The previous experiment is committed to studying the pharmacodynamic foundations by which S. officinalis L. affects platelet production, which has found that the total saponins of S. officinalis L. can increase the white blood cell and platelet counts [7]. The pentacyclic triterpenes, such as ziyuglycoside I, and ellagic acids, such as TMEA, DMAG, and DOMA (Figure 1), can increase platelet production by enhancing the proliferation and differentiation of the megakaryotic progenitor cells. The ellagic acids are known to be much more potent than the pentacyclic triterpenes, especially in promoting the megakaryocyte differentiation, a process related to the upregulation of hTPOR and hC-KIT mRNA [7, 8]. This lays an important foundation for the development of candidate drugs to promote platelet production.

Pharmacokinetics focuses on the regulation of absorption, distribution, metabolism, and excretion of drugs in vivo. It provides a basis for the route, dosage, interval, and duration of drug administration, thus ensuring the safety and efficacy of drug therapy. However, the pharmacokinetics of TMEA from S. officinalis L. in rats has not been reported, and its compatibility with different drugs may also cause changes in its pharmacokinetic parameters. UHPLC-MS/MS can avoid the complicated and cumbersome sample pretreatment steps to save the time of separation and purification, as well as increase the peak capacity, shorten the analysis time, and reduce the interference of the endogenous substance $[9,10]$. It has been gradually applied to pharmacokinetics, metabolomics, proteomics, drug residues, and so on [11]. It is now the most efficient method for the analysis and identification of trace components in biological samples because of its high sensitivity and selectivity. For example, Deng et al. [12] established and verified a simple and sensitive UHPLC-MS/MS method for the analysis of furandienone in rat plasma. Huang et al. [13] have reported a UHPLC-MS/MS method for the determination of kurarinone and its preclinical pharmacokinetic study in dogs after oral administration. A UHPLC-MS/MS method has been successfully applied to explore the oral and intravenous pharmacokinetics of skimmin [14]. Therefore, for the first time, this study aims to establish an efficient and sensitive UHPLC-MS/MS method to study the pharmacokinetic characteristics of TMEA after its administration in rats. It also aims to compare the pharmacokinetic changes between the normal and thrombocytopenic samples and those among the groups with and without compatibility. At the same time, the efficacy and safety of TMEA for the treatment of thrombocytopenia can be evaluated, which provides a scientific basis for further in-depth research and development of candidate drugs for thrombocytopenia.

\section{Materials and Methods}

\subsection{Materials}

2.1.1. Chemicals and Reagents. TMEA (BBP00642, purity $>98 \%$ ), DMAG (BBP03490, purity $>98 \%$ ), and DOMA (purity $>98 \%$ ) were obtained from BioBioPha Co., Ltd. (Yunnan, China). Artemetin (MUST-16090508, purity $>98 \%$, internal standard, IS) was supplied by Chengdu Mansite Pharmacetical Co., Ltd. (Chengdu, China). Cyclophosphamide (M17787, purity $>98 \%$ ) was purchased from Meryer Chemical Technology Co., Ltd. (Shanghai, China). Ethyl acetate was purchased from Aladdin Reagents Co., Ltd. (Shanghai, China). LC/MS grade formic acid (T-27563) and acetonitrile were purchased from Thermo Fisher Scientific Co., Ltd. (USA). TIANamp Genomic DNA Kit was purchased from Tiangen Biotech Co., Ltd. (Beijing, China). Quick Start Bradford 1x Dye Kit was purchased from Bio-Rad Laboratories (California, USA). Distilled deionized water was freshly generated by a Plus Milli-Q water purification system (Bedford, MA, USA). 
<smiles>COc1cc2oc(=O)c3cc(O)c(OC)c4oc(=O)c(c1)c2c43</smiles>

(a)<smiles>COc1ccc(C2Oc3cc(OC)c(OC)c(O)c3C(=O)C2OC)cc1OC</smiles>

(b)<smiles>COc1c(O)cc2c(=O)oc3c(OC)c(O[C@@H]4O[C@H](CO)[C@@H](O)[C@H](O)[C@H]4O)cc4c(=O)oc1c2c34</smiles>

(c)<smiles>COc1cc2c(=O)oc3c(OC)c(O[C@@H]4OC[C@@H](O)[C@H](O)[C@H]4O)cc4c(=O)oc(c1OC)c2c34</smiles>

(d)

Figure 1: The chemical structures of TMEA (a), IS (b), DMAG (c) and DOMA (d).

2.1.2. Animal Care. Sprague Dawley rats (certificate number: SCXK2020-030, 8 to 10 weeks old, bodyweight of 180 to $220 \mathrm{~g}$, half male and half female) were obtained from Da-suo Bio-technology Co., Ltd. (Chengdu, China). All animal experiments were performed in strict accordance with the U. S. guidelines [15] that were approved by the ethical committee on Use and Care of Animals of Southwest Medical University (No. 20160276). Prior to the experiment, animals were housed in a temperaturecontrolled facility with a 12-h light/dark cycle and were provided unlimited access to food and water for 7 days. The animals were subsequently fasted for $12 \mathrm{~h}$ before dosing, with free access to water.

\subsection{Methods}

2.2.1. Establishment of the Thrombocytopenia Rat Model. A thrombocytopenic rat model was established according to the method in relevant literature [16]. Following this method, 40 healthy SD rats (half male and half female) were arbitrarily divided into the normal $(n=12)$ and model groups $(n=28)$. The model group was intraperitoneally injected with $150 \mathrm{mg} / \mathrm{kg}$ cyclophosphamide on the first day and $50 \mathrm{mg} / \mathrm{kg}$ cyclophosphamide daily from the days 2 to 3 . A solution of $0.9 \%$ physiological saline was intraperitoneally injected into the normal group using the same dosage. On the last day, $40-\mu \mathrm{L}$ blood samples were collected from six normal rats and six model rats. Routine blood tests were subsequently performed using an XT-1800i/2000IV automatic blood cell analyzer (Sysmex Corp., Kobe, Japan). The bone marrow cells were collected, and the total bone marrow cell count, DNA content, and protein content of each sample were calculated with the automatic blood cell analyzer, a
NanoDrop OneC microultraviolet-visible spectrophoto meter (Thermo Electron Corp., Waltham, USA), and a BioTek Cytation3 multifunctional cell imaging detection system (BioTek Corp., Vermont, USA), respectively. Simultaneously, the heart, liver, spleen, lung, kidney, and thymus were harvested to measure the visceral index.

2.2.2. Instrument and Chromatographic Conditions. The analysis was performed on a UHPLC-MS/MS system (Shimadzu Corp., Tokyo, Japan) using an Acquity Shimpack GIST column $(2.0 \times 75 \mathrm{~mm}, 2.0 \mu \mathrm{m}$, Shimadzu Corp., Japan), consisting of a SIL-30AC automatic sampling device, four LC-30AD infusion pumps, a DGU-20A5 online degasser, an SPD-M30A detector, an LC-MS-8050 triplequadrupole mass spectrometer, a CTO20A column oven, and a CBM-20A system controller. The column temperature was maintained at $40^{\circ} \mathrm{C}$ with a flow rate of $0.3 \mathrm{~mL} / \mathrm{min}$. The mobile phase for this analysis was composed of $0.1 \%$ formic acid water (solvent A) and acetonitrile (solvent B). The gradient condition was as follows: a linear gradient elution of $70 \%$ to $90 \%$ solvent B for the initial 2 min, followed by an isocratic elution of solvent $B$ for the next. The injection volume was $10 \mu \mathrm{L}$.

The mass spectrometer was operated in the positive and negative ion mode. Multiple reaction monitoring (MRM) acquisition mode was used to monitor the precursor-toproduct ion transition. The MRM fragmentation transitions were $\mathrm{m} / \mathrm{z} \quad 343.20 \longrightarrow 328.10$ for TMEA and $\mathrm{m} / \mathrm{z}$ $389.15 \longrightarrow 331.15$ for IS (Figure 2). The collision energy for TMEA and IS were set at $17 \mathrm{eV}$ and $35 \mathrm{eV}$, respectively. The flow rates of atomizing gas, heating gas, and drying gas were $2.0 \mathrm{~L} / \mathrm{min}, 10.0 \mathrm{~L} / \mathrm{min}$, and $10.0 \mathrm{~L} / \mathrm{min}$, respectively. The interface voltage and temperature were maintained at $4 \mathrm{kV}$ and 


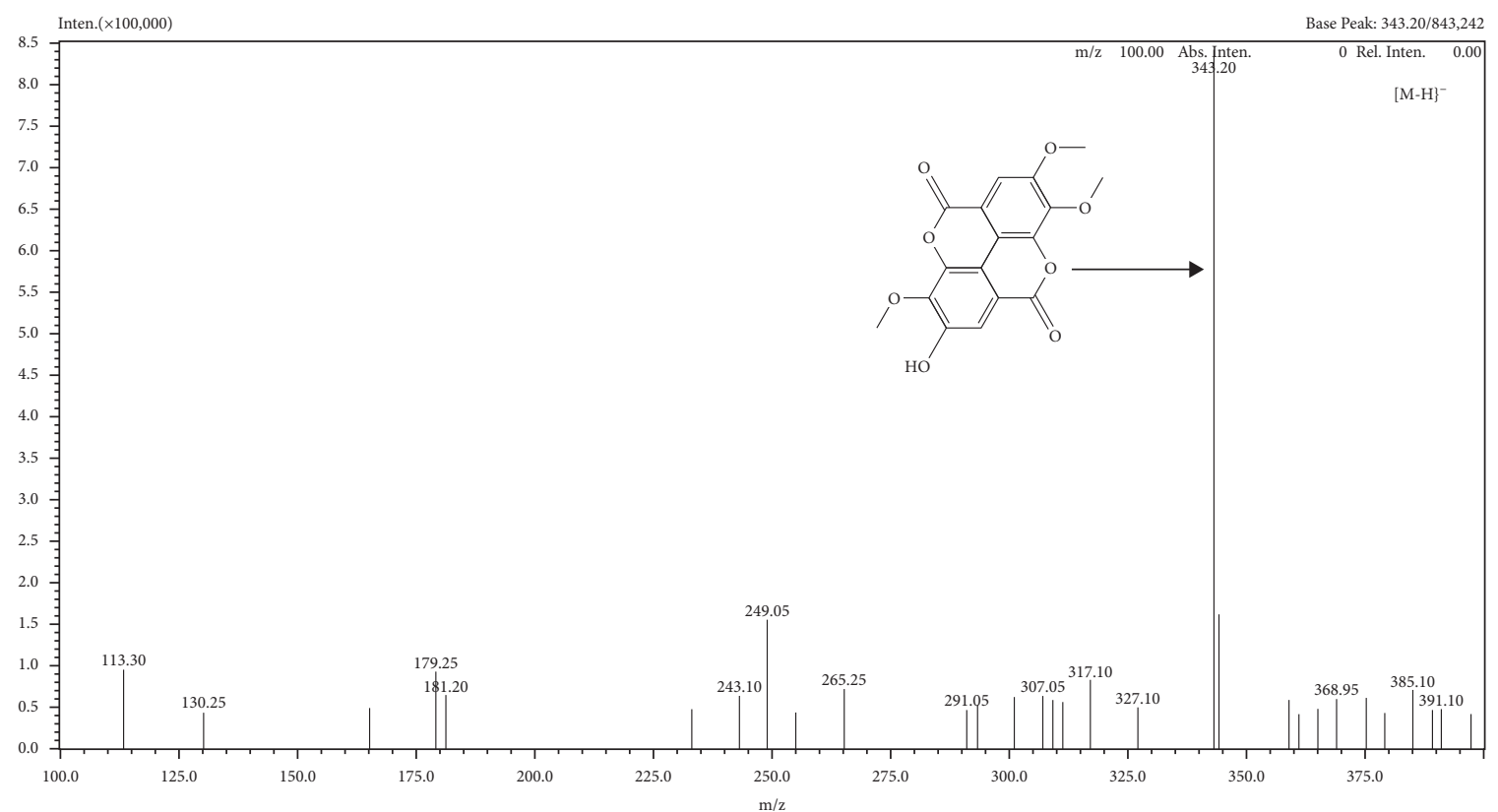

(a)

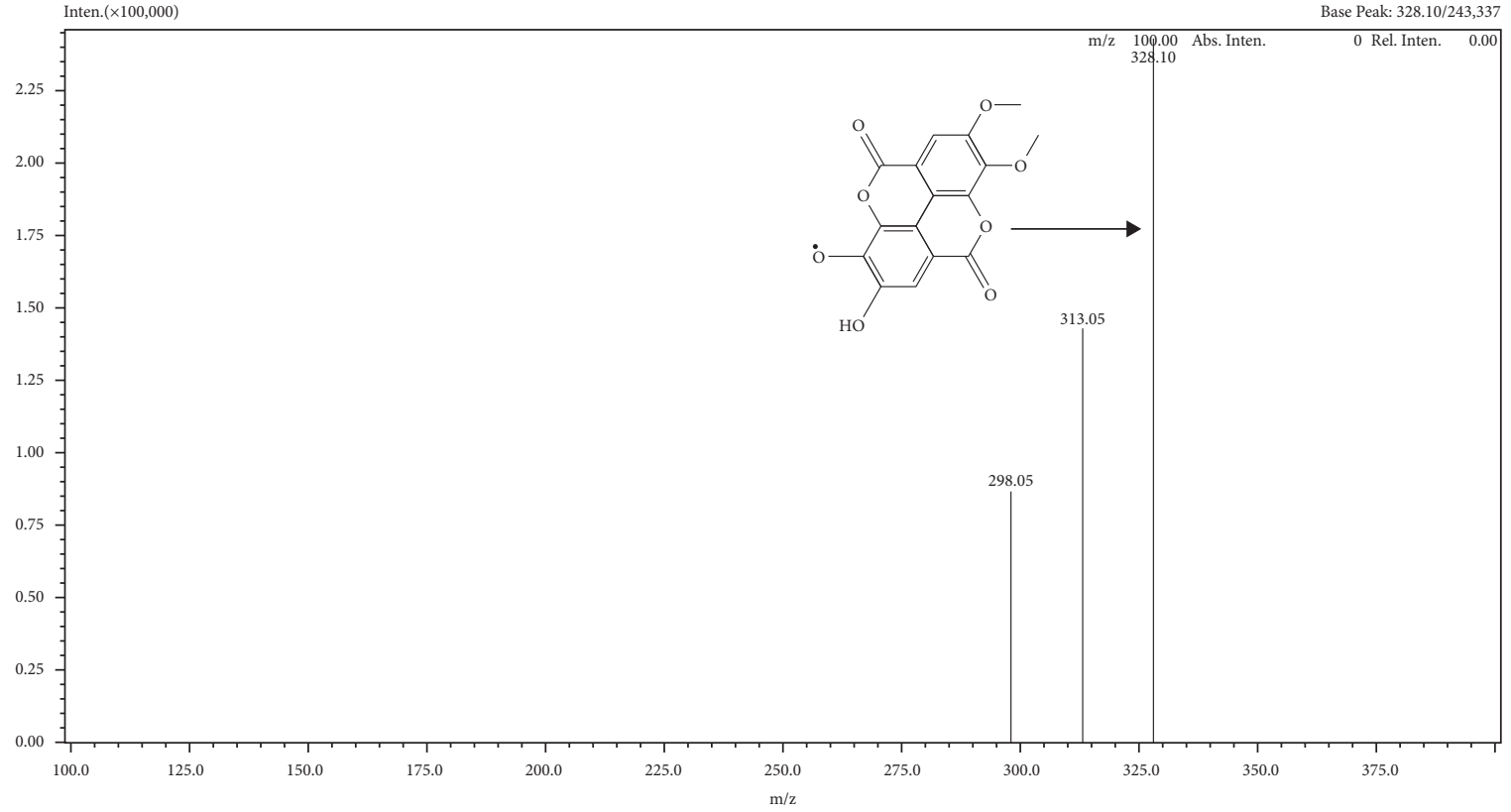

(b)

FIgUre 2: Continued. 


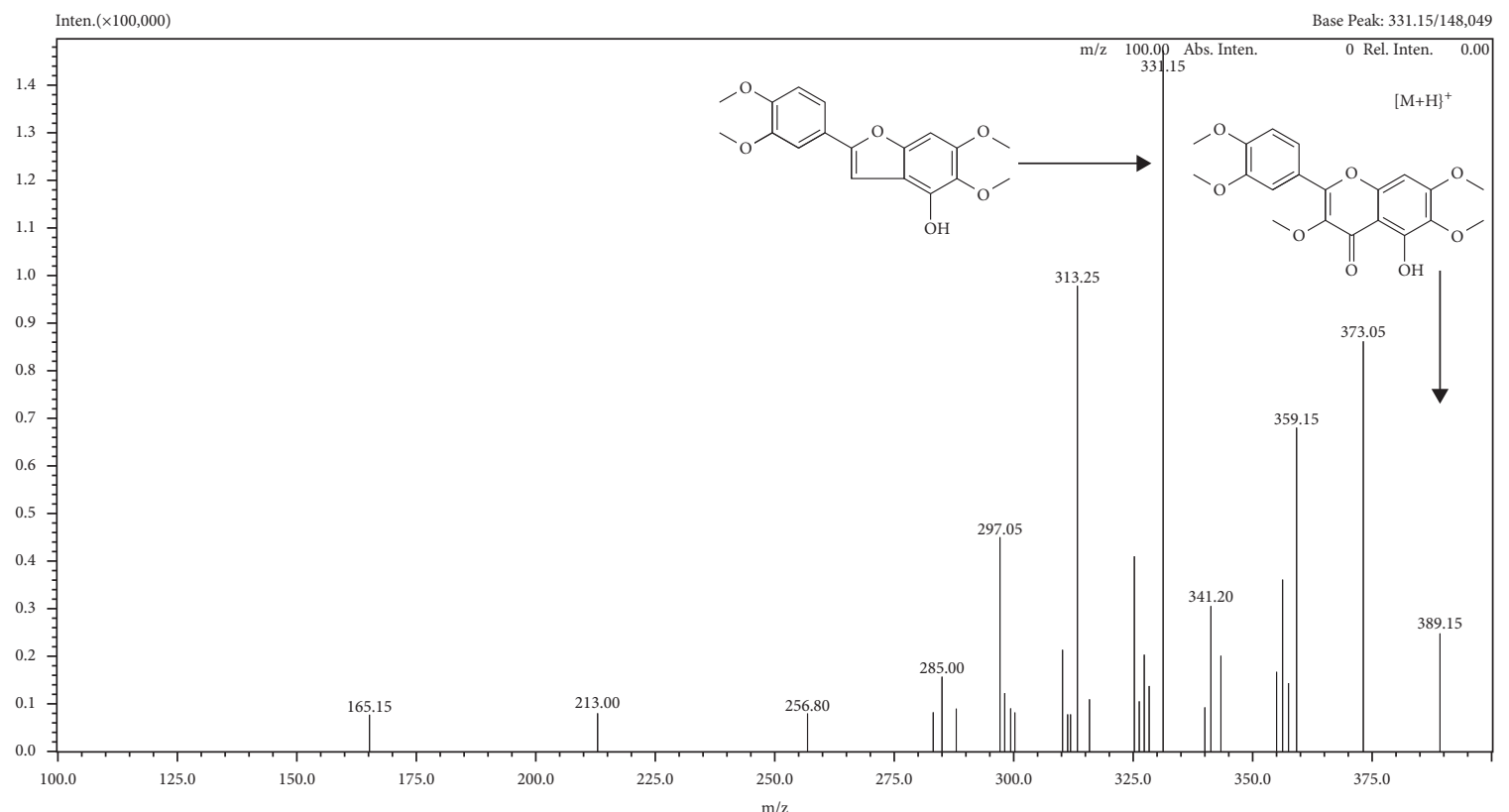

(c)

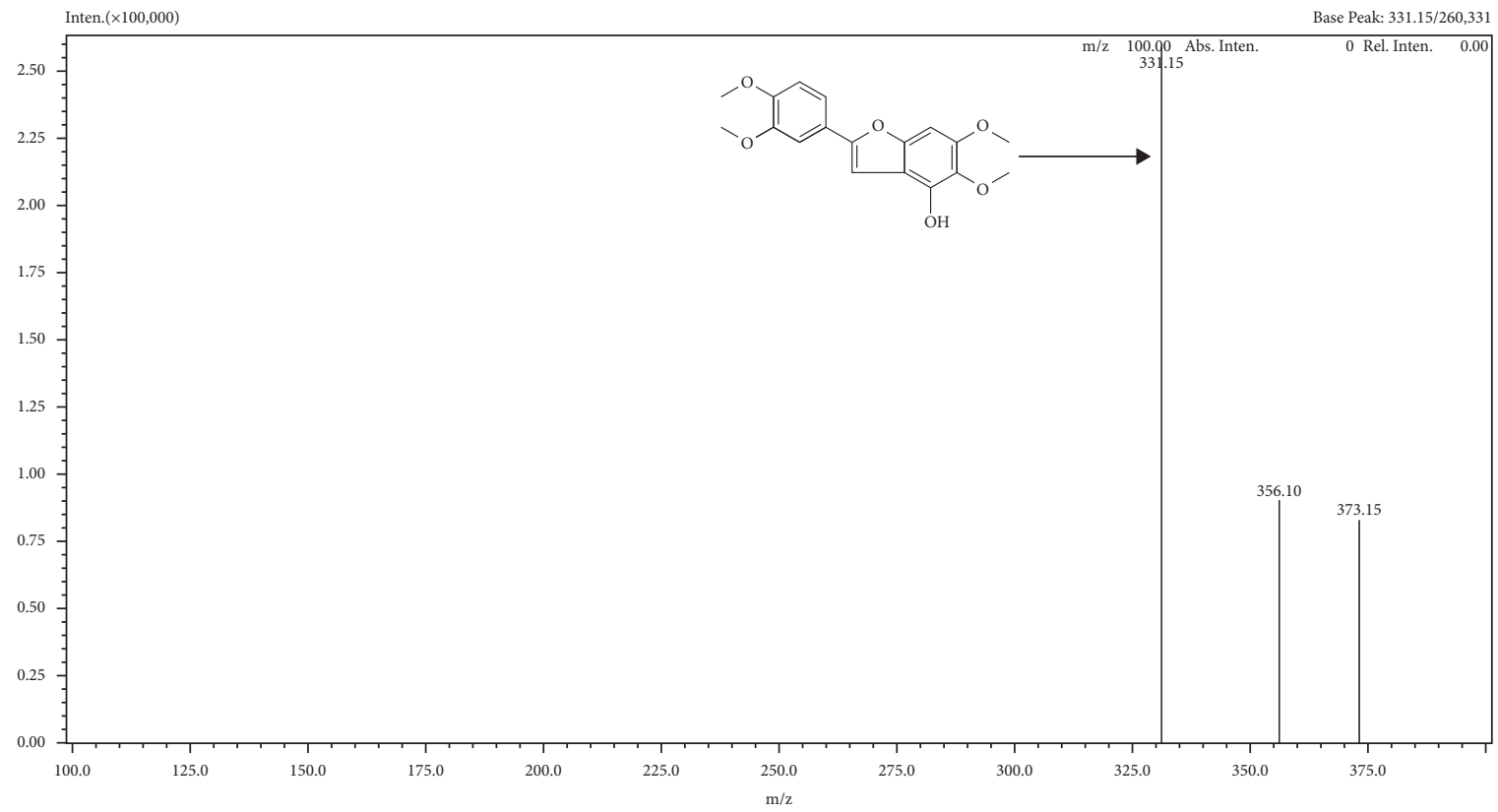

(d)

Figure 2: The MS/MS spectrums of TMEA (a) and IS (c), and the main corresponding product ion of TMEA (b) and IS (d).

$300^{\circ} \mathrm{C}$. The desolvation temperature was $250^{\circ} \mathrm{C}$, heating block temperature was $400^{\circ} \mathrm{C}$, and detector voltage was $2.24 \mathrm{kV}$.

2.2.3. Preparation of Samples. The stock solutions of TMEA and IS were prepared with acetonitrile at 0.051 and $19.41 \mathrm{mg} /$ $\mathrm{mL}$, respectively. The working standard solutions of TMEA were prepared by serially diluting a stock solution to achieve a series of concentrations: $5,10,50,100,500,1000,4000 \mathrm{ng} / \mathrm{mL}$ and IS stock solutions were diluted into $500 \mathrm{ng} / \mathrm{mL}$. The solutions were stored for no longer than 14 days at $-20^{\circ} \mathrm{C}$. Standard samples were prepared by spiking appropriate amounts of the stock solutions of TMEA and IS in blank plasma. The quality control samples for assessing the method accuracy and precision were independently prepared at low (1 ng/mL), medium (10 ng/mL), and high (100 ng/mL) concentrations in the same manner. After $100 \mu \mathrm{L}$ plasma samples were spiked with $10 \mu \mathrm{L}$ IS solution and mixed for $30 \mathrm{~s}, 2 \mathrm{~mL}$ ethyl acetate was added to each tube. The tubes were vortexed for $5 \mathrm{~min}$ and left to stand for $1 \mathrm{~min}$ at room temperature. The extracts were centrifuged at $8000 \mathrm{~g}$ for $10 \mathrm{~min}$ at $4^{\circ} \mathrm{C}$. The supernatant was dried under a gentle stream of nitrogen at $37^{\circ} \mathrm{C}$. The extract residues were reconstituted, and $10 \mu \mathrm{L}$ samples were subsequently injected for analysis. 
2.2.4. Method Validation. The method was validated in terms of specificity, linearity, matrix effect, recovery, accuracy, precision, and stability in accordance with Food and Drug Administration (FDA) guidelines [17]. Specificity was evaluated by comparing the chromatograms of blank plasma, blank plasma spiked with TMEA and IS, and plasma collected after the oral administration of TMEA. The calibration curve was generated by plotting the peak-area ratio of TMEA and IS with nominated concentrations. Three concentrations of the quality control (QC) samples $(1,10$, and $100 \mathrm{ng} / \mathrm{mL}$ ) were analyzed in six replicates on the same day and on three consecutive days to evaluate the intraday and interday precision and accuracy. Accuracy was expressed as the relative error between the spiked and determined concentrations, while precision was presented as a relative standard deviation (RSD). The matrix effect and recovery were calculated as the peak area of TMEA with or without plasma and extraction with ethyl acetate. The stability of TMEA in rat plasma was evaluated in different storage and handling conditions with all three QC sample concentrations.

2.2.5. Pharmacokinetic Study. Eighteen thrombocytopenia rats were arbitrarily divided into three groups, which are the TMEA $(n=6)$, TMEA-DMAG $(n=6)$, and TMEA-DOMA $(n=6)$ group. $4 \mathrm{mg} / \mathrm{kg}$ TMEA was intragastrically administered to the TMEA group. $4 \mathrm{mg} / \mathrm{kg}$ TMEA and $4 \mathrm{mg} / \mathrm{kg}$ DMAG were intragastrically administered to the TMEA-DMAG group, while $4 \mathrm{mg} / \mathrm{kg}$ TMEA and $4 \mathrm{mg} / \mathrm{kg}$ DOMA were orally administered to the TMEA-DOMA group simultaneously. The same dosage of TMEA was similarly administered to the normal group $(n=6)$. The blood samples were collected from the tail vein at $0,0.08$, $0.25,0.5,1,1.5,2,3,4,6,8,12,24,36,48$, and $72 \mathrm{~h}$ and stored in heparinized tubes. The plasma samples were obtained following centrifugation at $1500 \mathrm{~g}$ for $10 \mathrm{~min}$ and kept frozen at $-80^{\circ} \mathrm{C}$ until analysis.

2.3. Statistical Analysis. The pharmacokinetic parameters were calculated using DAS 3.0. The T-test was performed using GraphPad Prism 8.0. The data were expressed as mean \pm standard error. A difference at $p<0.05$ was considered to be statistically significant.

\section{Results and Discussion}

3.1. Establishment of the Thrombocytopenia Rat Model. Ideal and appropriate animal models play a key role in drug development. The cyclophosphamide-induced thrombocytopenia rat model used in the study is a classical model in hematological research. Cyclophosphamide is a commonly used antitumor drug that also inhibits hematopoietic and immune functions. The platelet and bone marrow cell counts, DNA and protein content, and spleen and thymus index of the model rats were all found to be significantly decreased compared to normal rats (Figure 3), indicating that the thrombocytopenia rat model was successfully established.
3.2. Optimization of Determination Conditions. Firstly, a rapid, sensitive, and specific method to detect TMEA was established in this study. The use of formic acid contributed to an enhanced ionization efficiency and a stabler baseline (Figure 4). To improve the peak shape and retention time, $0.1 \%$ formic acid water and acetonitrile were chosen as the mobile phase. In this experiment, acetone, ethyl ether, dichloromethane, chloroform, ethyl acetate, and other solvents were used to investigate the extraction efficiency of TMEA. The results showed that the recovery of acetone, ethyl ether, dichloromethane, and chloroform was much lower than that of ethyl acetate. Hence, ethyl acetate was selected as the extraction solvent (Table 1).

3.3. Method Validation. The specificity of the method was investigated by analyzing the rat plasma from different sources. The typical chromatograms of blank plasma, blank plasma spiked with TMEA and IS, and plasma collected after the oral administration of TMEA are shown in Figure 5. The retention times of TMEA and IS were 1.183 and $1.530 \mathrm{~min}$, respectively. The chromatograms showed no endogenous peaks interfering with the detection of TMEA and IS, establishing the excellent specificity of this method. The calibration curve of TMEA was fitted to linear regression in the concentration range of 0.5 to $400.0 \mathrm{ng} / \mathrm{mL}$. The linear equation was $y=0.013752 x+0.006824\left(r^{2}=0.9996\right)$. The lower limit of quantification (LLOQ) was defined as $0.5 \mathrm{ng} /$ $\mathrm{mL}$ in the study. The intraassay and interassay precision for TMEA is shown in Table 2. The intraday RSD was $<9.44 \%$, interday was RSD $<9.50 \%$, and its relative error of accuracy was between $-5.70 \%$ and $2.96 \%$ in rat plasma. The recovery and matrix effect of TMEA at three QC concentrations are enumerated in Table 3, the former was between $94.77 \%$ and $102.47 \%$ and the latter was between $93.16 \%$ and $100.15 \%$. As shown in Table 4, the stability of TMEA in different storage conditions contained at $25^{\circ} \mathrm{C}$ for $12 \mathrm{~h}, 4^{\circ} \mathrm{C}$ for $24 \mathrm{~h},-80^{\circ} \mathrm{C}$ for one month, and three freeze-thaw cycles was evaluated at 1 , 10 , and $100 \mathrm{ng} / \mathrm{mL}$. These results indicate stability in the rat samples during the preparation and analytical processes, with the stability of RSD between $-8.55 \%$ and $2.98 \%$. These findings suggest that the method was sufficiently sensitive for investigating the pharmacokinetics of TMEA in vivo.

3.4. Pharmacokinetic Study. As shown in Table 5 and Figure 6 , with a remarkably decreased $T_{\max }$ and $\mathrm{CL} / \mathrm{F}$, the values of $\mathrm{AUC}_{(0-t)}, \mathrm{AUC}_{(0-\infty)}$, and $C_{\max }$ of TMEA in the TMEA group were clearly higher than those in the normal group. The results showed that the pharmacokinetics of TMEA differed between the normal and thrombocytopenia rats, which indicated that the pharmacokinetic changes may be caused by the pathological conditions. A similar phenomenon has also been observed in relevant literature. Zhao et al. [18] have reported that the $C_{\max }$ and AUC of genipin 1-gentiobioside, geniposide, naringenine-7-rhamnosidoglucoside, hesperidin, and neohesperidin in the depression rats increased significantly compared to the normal rats after the administration of ZhiShi-Zhi-Zi-Chi decoction. Hui et al. [19] found that there were significant differences in AUC, mean residence time (MRT), 


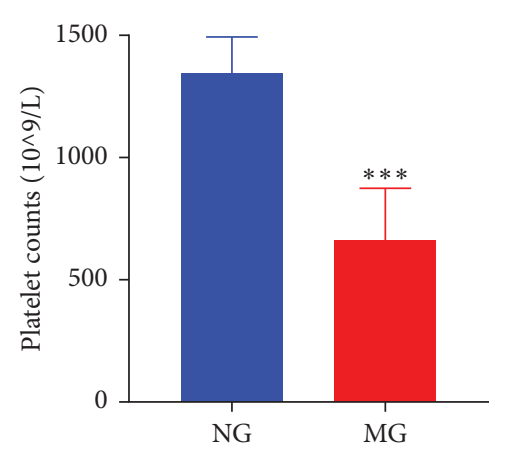

(a)

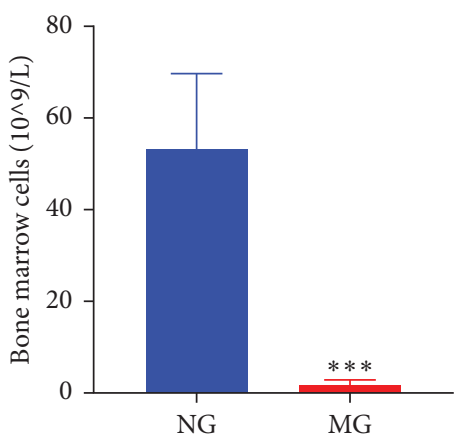

(b)

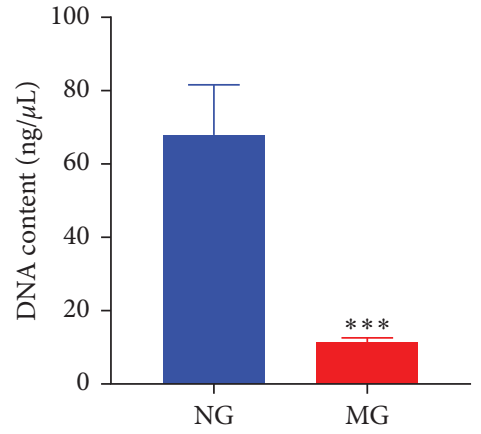

(c)

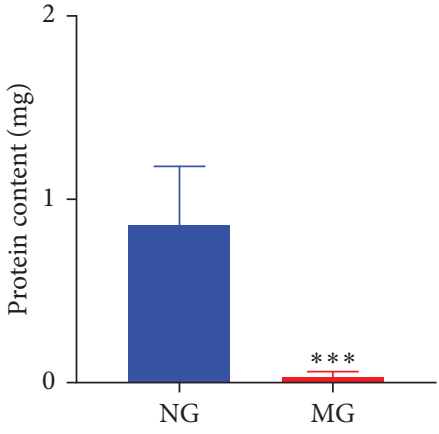

(d)

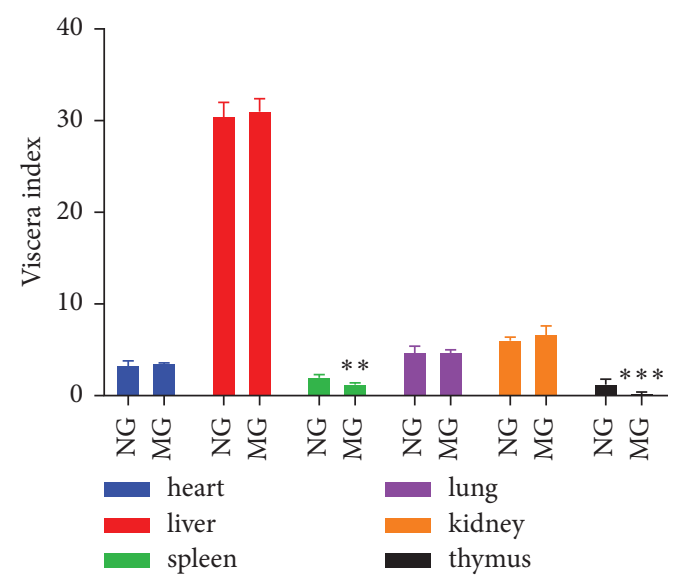

(e)

Figure 3: The platelet count (a), bone marrow cell count (b), DNA content (c), protein content (d) and visceral index (e) of the normal and model rats. Normal group and model group are abbreviated to NG and MG, respectively. ${ }^{* *} P<0.01$, ${ }^{* * *} P<0.01$ significant compared with NG.
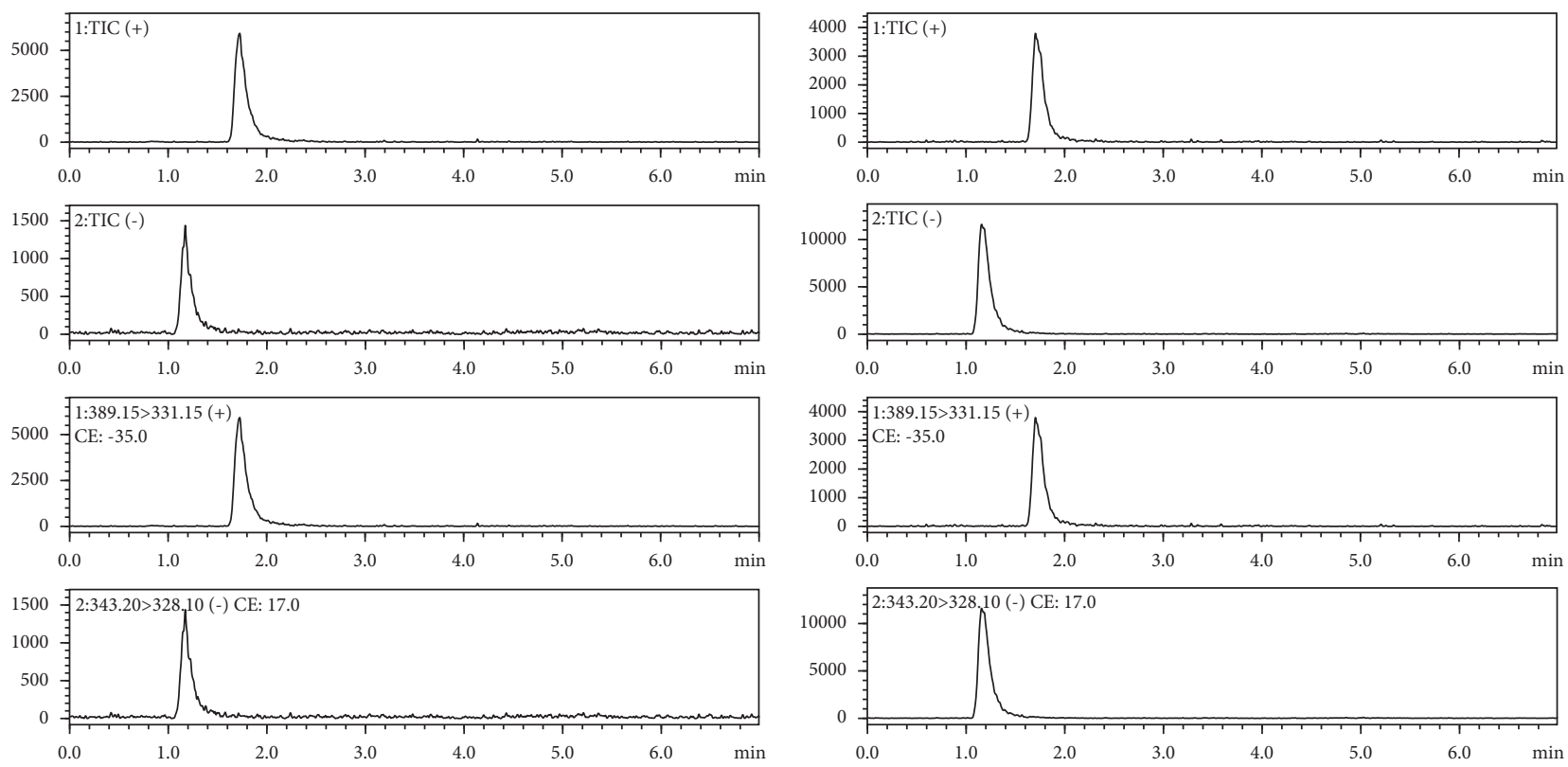

(a)

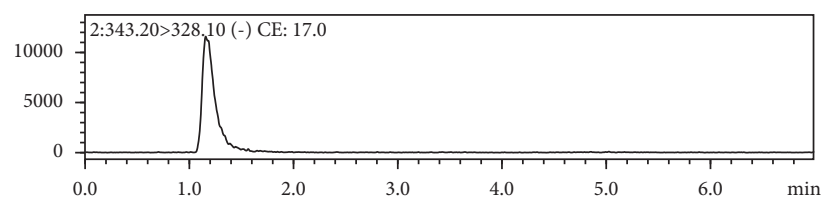

(b)

FIGURE 4: The MRM chromatograms of standard solution with $1 \mathrm{ng} / \mathrm{mL}$ TMEA and IS in pure water and acetonitrile (a) and $0.1 \%$ formic acid water and acetonitrile (b). 
TABLE 1: Recovery of TMEA with different extraction solvents $(\%, n=6)$.

\begin{tabular}{lcccr}
\hline acetone & Ethyl ether & Dichloromethane & Chloroform & Ethyl acetate \\
\hline $53.75 \pm 6.26$ & $6.69 \pm 2.29$ & $54.04 \pm 12.72$ & $41.89 \pm 7.62$ & $100.86 \pm 6.79$ \\
\hline
\end{tabular}
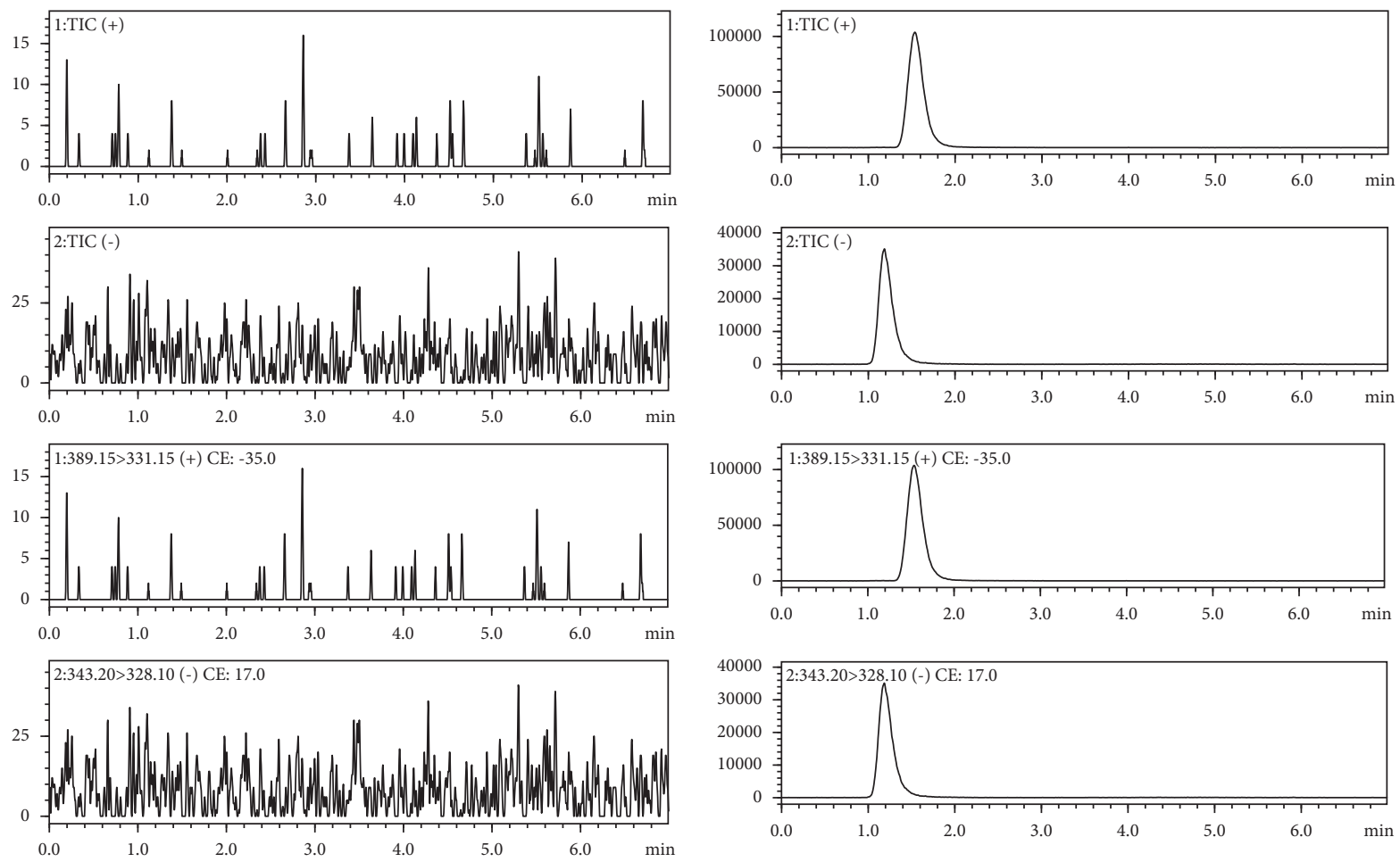

(a)

(b)
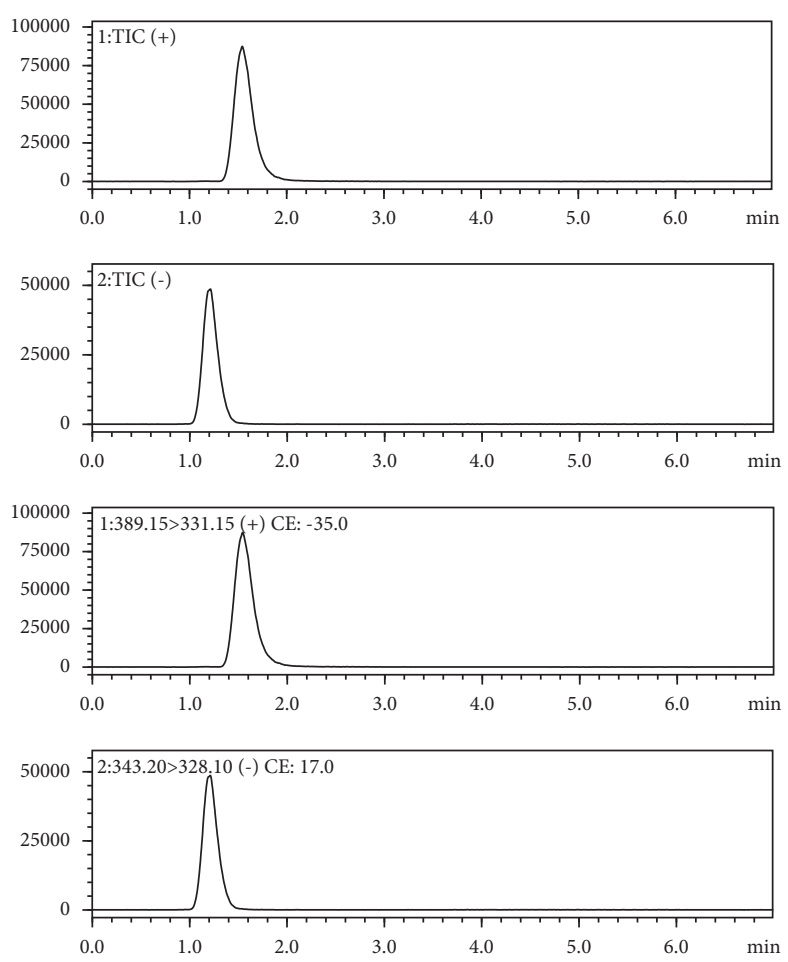

(c)

Figure 5: The MRM chromatograms of blank plasma (a), blank plasma with $5 \mathrm{ng} / \mathrm{mL}$ TMEA and $50 \mathrm{ng} / \mathrm{mL}$ IS (b), and plasma collected at $0.5 \mathrm{~h}$ after treated with $4 \mathrm{mg} / \mathrm{kg}$ TMEA in thrombocytopenia rat and $50 \mathrm{ng} / \mathrm{mL}$ IS (c). 
TABLE 2: Precision and accuracy for intra-day and inter-day of TMEA $(n=6)$.

\begin{tabular}{|c|c|c|c|c|c|c|}
\hline \multirow{2}{*}{ Concentration $(\mathrm{ng} / \mathrm{mL})$} & \multicolumn{3}{|c|}{ Intra-day } & \multicolumn{3}{|c|}{ Inter-day } \\
\hline & Mean \pm SD & Accuracy (RE\%) & Precision (RSD\%) & Mean \pm SD & Accuracy (RE\%) & Precision (RSD\%) \\
\hline 1.00 & $0.99 \pm 0.05$ & -0.46 & 5.75 & $0.95 \pm 0.03$ & -4.80 & 3.89 \\
\hline 10.00 & $10.29 \pm 0.97$ & 2.96 & 9.44 & $9.42 \pm 0.89$ & -5.70 & 9.50 \\
\hline 100.00 & $100.17 \pm 8.95$ & 0.17 & 8.93 & $98.00 \pm 8.31$ & -1.99 & 8.47 \\
\hline
\end{tabular}

TABLE 3: Recovery and matrix effect of TMEA $(\%, n=6)$.

\begin{tabular}{lcr}
\hline Concentration $(\mathrm{ng} / \mathrm{mL})$ & Recovery & Matrix effect \\
\hline 1.00 & $99.81 \pm 5.90$ & $93.16 \pm 5.42$ \\
10.00 & $94.77 \pm 8.38$ & $98.92 \pm 7.66$ \\
100.00 & $102.47 \pm 8.04$ & $100.15 \pm 9.05$ \\
\hline
\end{tabular}

TABLE 4: Stability of TMEA under different storage condition (RSD $\%, n=6)$.

\begin{tabular}{lcccc}
\hline Concentration $(\mathrm{ng} / \mathrm{mL})$ & Short-term $\left(12 \mathrm{~h}, 25^{\circ} \mathrm{C}\right)$ & Plasma sample $\left(24 \mathrm{~h}, 4^{\circ} \mathrm{C}\right)$ & Long-term $\left(30 \mathrm{~d},-80^{\circ} \mathrm{C}\right)$ & Three freeze-thaw cycles $\left(-80^{\circ} \mathrm{C}\right)$ \\
\hline 1.00 & -8.55 & -1.27 & 0.28 & -7.34 \\
10.00 & -7.26 & 2.98 & 0.60 & -2.58 \\
100.00 & 1.95 & 1.08 & -4.54 & -6.57 \\
\hline
\end{tabular}

TABLE 5: Pharmacokinetic parameter of TMEA in rat after intragastric administration of four groups $(n=6)$.

\begin{tabular}{lcccc}
\hline Parameter & NG & TG & TMG & TOG \\
\hline AUC $_{0-t}(\mathrm{ng} \cdot \mathrm{h} / \mathrm{mL})$ & $752.68 \pm 144.33$ & $1185.67 \pm 178.36^{* *}$ & $886.47 \pm 190.72^{\mathbf{\Lambda}}$ & $1895.37 \pm 286.73^{\mathbf{\Lambda}}$ \\
$\mathrm{AUC}_{0-\infty}(\mathrm{ng} \cdot \mathrm{h} / \mathrm{mL})$ & $833.87 \pm 169.63$ & $1462.82 \pm 346.33^{* *}$ & $1121.94 \pm 188.25$ & $3023.54 \pm 983.08^{\mathbf{\Lambda}}$ \\
MRT $(\mathrm{h})$ & $28.85 \pm 12.25$ & $40.15 \pm 10.22$ & $45.53 \pm 23.66$ & $78.70 \pm 58.83$ \\
$t_{1 / 2}(\mathrm{~h})$ & $23.61 \pm 16.09$ & $34.80 \pm 14.36$ & $36.50 \pm 19.41$ & $61.43 \pm 45.40$ \\
$T_{\max }(\mathrm{h})$ & $3.16 \pm 0.40$ & $2.16 \pm 0.40^{* *}$ & $3.00 \pm 0.00^{\mathbf{\Lambda}}$ & $2.00 \pm 0.00$ \\
$V_{\mathrm{z}} / F(\mathrm{~mL} / \mathrm{kg})$ & $160604.50 \pm 89246.64$ & $133606.50 \pm 23554.64$ & $186729.66 \pm 84733.41$ & $109132.16 \pm 49342.68$ \\
$\mathrm{CL} / \mathrm{F}(\mathrm{mL} / \mathrm{h} / \mathrm{kg})$ & $4950.33 \pm 913.25$ & $2845.83 \pm 570.44^{* *}$ & $3652.00 \pm 628.26^{\mathbf{\Delta}}$ & $1412.33 \pm 337.40^{\mathbf{\Lambda}}$ \\
$C_{\max }(\mathrm{ng} / \mathrm{mL})$ & $68.03 \pm 10.75$ & $115.92 \pm 14.53^{* *}$ & $89.92 \pm 5.34^{\mathbf{\Lambda}}$ & $198.36 \pm 51.53^{\mathbf{\Lambda}}$ \\
\hline
\end{tabular}

Abbreviations: NG, normal group; TG, TMEA group; TMG, TMEA-DMAG group; TOG, TMEA-DOMA group; AUC $_{0-t}$, area under the concentration-time curve from 0 to $72 \mathrm{~h} ; \mathrm{AUC}_{0-\infty}$, area under the concentration-time curve from 0 to time infinite; MRT, mean residence time; $t_{1 / 2}$, half-life of elimination; $T_{\max }$, time to achieve maximum concentration; $V_{z} / F$, volume of distribution; CL/F, clearance; $C_{\max }$, maximum value of concentration; ${ }^{* *} P<0.01,{ }^{* * *} P<0.01$ significant compared with NG, ${ }^{\mathbf{\Delta}} P<0.05,{ }^{\boldsymbol{\Delta}} \mathrm{P}<0.01$ compared with TG.

$\mathrm{CL} / \mathrm{F}$, and the apparent volume of distribution $\left(V_{\mathrm{z}} / F\right)$ between the normal and arthritis rats for the five active components of Periploca forrestii Schltr (P. forrestii): neochlorogenic acid, chlorogenic acid, cryptochlorogenic acid, isochlorogenic acid $\mathrm{C}$, and periplocin. Compared to cerebral ischemia rats, the CL/ $\mathrm{F}$ value of tanshinol, hydroxysafflor yellow $\mathrm{A}$, and salvianolic acid A and B was lower in the normal rats, although AUC was increased after the intravenous injection of Salvia miltiorrhiza Bge. and Carthamus tinctorius L. These findings might be related to the destruction of the blood-brain barrier [20]. Fan et al. [21] initially reported that the pharmacokinetics of thirteen phenolic acids and six triterpenoids were significantly different between the normal and leukopenia rats after the oral administration of $S$. officinalis L. extract. In chemotherapyinduced thrombocytopenia rats, the $C_{\max }$ and terminal half-life $\left(\mathrm{T}_{1 / 2}\right)$ values for the H12-(ADP)-liposomes were decreased, however, CL/F was increased compared to the normal rats [22].

The intestinal flora may be altered in pathological conditions, potentially affecting drug metabolism. The intestinal tract is considered to be the largest immune organ in vivo and is the center of numerous immunoregulatory molecules that act to resist an invasion by foreign pathogens and maintain the stability of the human internal environment. A clinical study found that most patients with immune thrombocytopenia who also have intestinal infections or gastrointestinal dysfunction presented improved platelet counts after the alteration of intestinal flora [23]. At the moment, it is generally accepted that the imbalances in the intestinal flora may be highly correlated with the occurrence and development of immune thrombocytopenia. Gallotannins from S. officinalis L. can be hydrolyzed to an ellagic acid, which can be further metabolized by the intestinal flora into urolitins, such as urolitin A, urolitin B, urolitin C, urolitin D, and urolitin E [24]. It is possible that thrombocytopenia can be cured by regulating the intestinal flora after administering TMEA, DMAG, and DOMA. However, further study is needed to test this hypothesis. 

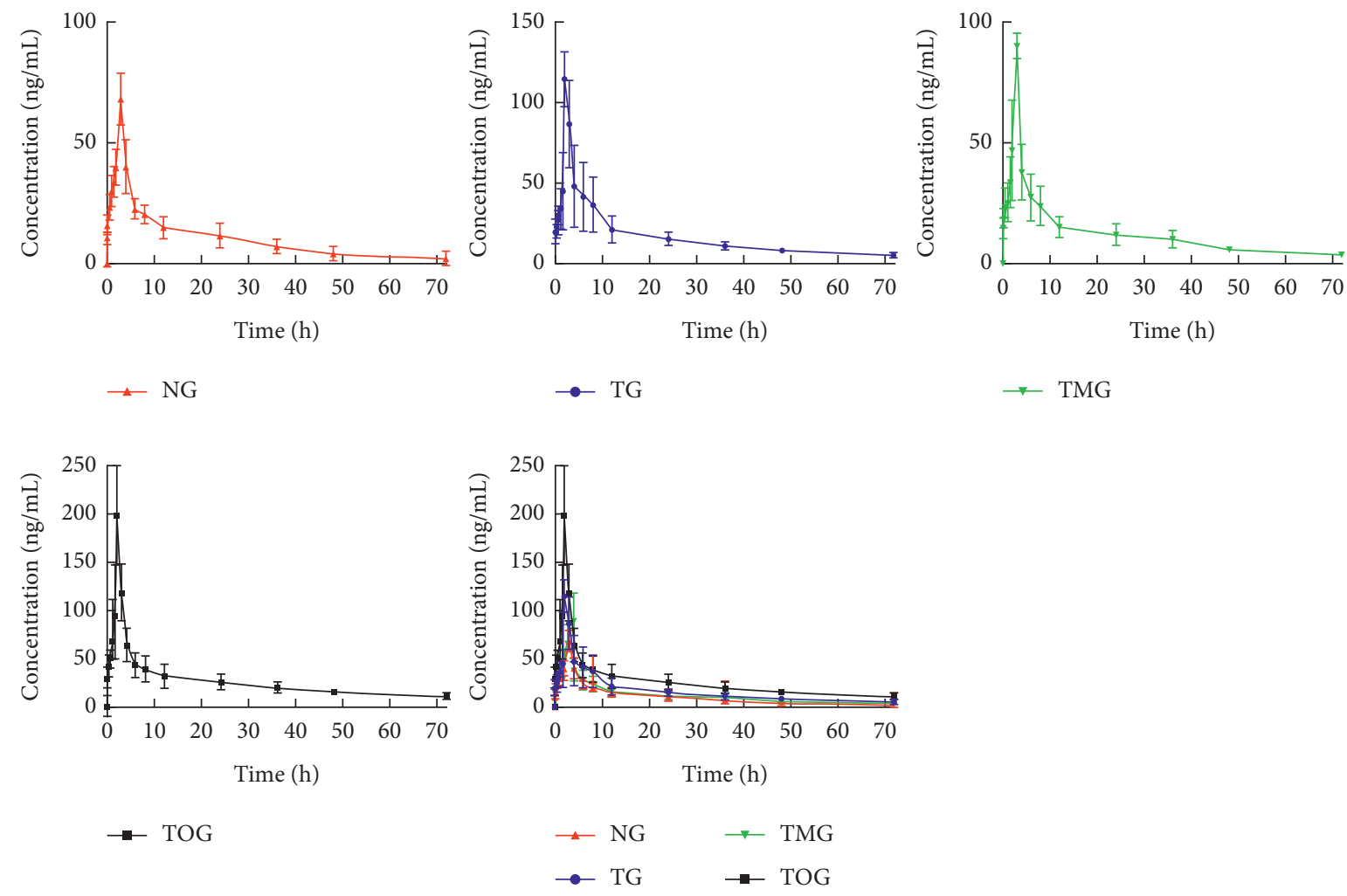

FIGURE 6: Mean plasma concentration-time profiles of TMEA after oral administration in rat of four groups $(n=6)$. Normal group, TMEA group, TMEA-DMAG group, TMEA-DOMA group are abbreviated to NG, TG, TMG and TOG, respectively.

The $\operatorname{AUC}_{(0-t)}, \mathrm{AUC}_{(0-\infty)}$, and $C_{\max }$ values for TMEA in the TMEA-DOMA group were nearly two times those of the TMEA group, while the CL/F value was remarkably reduced compared with the TMEA group. Unlike with DOMA, the $\mathrm{AUC}_{(0-t)}$ and $C_{\max }$ values were significantly decreased for TMEA with clearly prolonged $T_{\max }$ and increased $\mathrm{CL} / \mathrm{F}$ after combination with DMAG (Table 5 and Figure 6). The results indicated that the pharmacokinetics of TMEA were altered after compatibility with DMAG or DOMA, which suggested that the pharmacokinetic changes may also be caused by compatibility. This phenomenon has been found in literature. According to the past research, $T_{\max }$ was increased while $C_{\max }$ and AUC were decreased for albiflorin in the extracts of Danggui Shaoyao powder [25]. Liu et al. found that the pharmacokinetic parameters and brain distribution of magnolol and honokiol from the extracts of Magnolia officinalis Rehd. et Wils. were altered after combination with Gardenia jasminoides Ellis. and Citrus aurantium L. in Zhi$\mathrm{Zi}-\mathrm{Hou}$-Po decoction [26]. For example, $V_{\mathrm{z}} / F$ and CL/F of catechin, epicatechin, and ziyuglycoside I were significantly reduced, while the $T_{\max }$ value of ziyuglycoside I was lengthened with an accessorial $C_{\max }$ alongside an increase in the proportion of tannins [27]. The pharmacokinetic properties of typhylside, vanillic acid, and p-coumaric acid in Typha angustifolia L. were also altered after the compatibility with the components of trogopterus dung [28].

Compatibility may have an impact on the content and activity of the drug metabolizing enzymes. The liver is the primary metabolic organ, and the liver microsomes house most metabolic enzymes, including cytochrome P450 (CYP450), monoamine oxidase (Mao), lipase, glucuronidase (UGT), and glutathione transferase (GST). CYP450 levels have been shown to significantly decrease upon the administration of tannins from Rubus chingii $\mathrm{Hu}$ [29]. The GST activity was also found to be inhibited by the ellagic acid in a noncompetitive manner, suggesting that the ellagic acid may affect its active site [30]. These studies imply that the pharmacokinetics of TMEA in rats could be affected by DOMA, which is a similar kind of ellagic acid, by way of inhibiting CYP450 expression and GST activity. At the same time, DOMA can be converted to TMEA with the help of microsomes by the loss of glycosyl. However, because of the different chemical structure of DMAG, it may quicken the metabolism of TMEA to reduce the absorption.

Compatibility may also affect the function of efflux proteins such as breast cancer resistance protein (BCRP), multidrug resistance-associated protein 2 (MRP2), and especially $\mathrm{P}$-glycoprotein (P-gp). The chemical composition of a herb is complex. There are several active components in each herb that may interact in vivo and directly affect the efficacy and toxicity. As the transport rates of the gallic acid and ellagic acid were changed with the addition of verapamil, some components of S. officinalis L. may be substrates of P-gp [31]. It suggests that the other components of $S$. officinalis L., such as TMEA and DOMA, may also be the substrates of P-gp, may competitively bind P-gp, 
and affect the pharmacokinetic parameters of TMEA. Nevertheless, DMAG may be not the substrate of P-gp, BCRP, and MRP2.

TPO/c-Mpl, janus kinase/signal transducers and the activators of transcription (JAK/STAT), phosphatidylinositol 3 kinase/protein kinase B (PI3K/Akt), and mitogenactivated protein kinase/extracellular regulated protein kinases (MEK/ERK) are involved in the main thrombopoietic pathway. Transcription factors such as GATA binding protein-1 (GATA-1), GATA binding protein-2 (GATA-2), runt-related transcription factor-1 (RUNX-1), nuclear factor erythroid-2 (NF-E2), friend of GATA-1 (FOG-1), and friend leukemia virus integration-1 (FLI-1) play key roles in the directional differentiation of the megakaryocyte progenitor cells as these are regulated by the aforementioned pathways and promote the target gene expression and megakaryocyte maturation into platelets [32]. In addition, long noncoding RNA imprinted maternally expressed the transcript (lncRNA H19), IncRNA cancer susceptibility candidate 2 (CASC2), IncRNA antisense RNA binding protein (ASRBM15), circular RNA mannosidase alpha class 1A member 2 (circRNA MAN1A2), platelet circRNA 4 (Plt-circR4), miRNA-10a, miRNA-17, miRNA-20, miRNA-106, miRNA124-3p, miRNA-125b, miRNA-126, and miRNA-150. Other noncoding RNAs are also involved in the regulation of megakaryocyte production and differentiation [33]. This study found that pathological conditions and compatibility can affect the pharmacokinetics of TMEA. However, the molecular pathways involved remain unclear and need further research. Meanwhile, drug safety is key during the development of new drugs, and zebrafish has become the preferred model for screening and evaluating drug toxicity. A zebrafish model will be used to further investigate drug distribution in various organs in vivo and further evaluate toxicity.

\section{Concluding Remarks}

In this paper, a rapid, sensitive, and specific UHPLC-MS/MS method for the determination of TMEA was established and successfully applied in pharmacokinetic research in a rat model for the first time. The results revealed that the pharmacokinetic characteristics of TMEA in rats were altered because of the pathological condition of thrombocytopenia and because of compatibility. These findings provide valuable information for future research into $S$. officinalis L., and serve as guideline for the clinical development of new drugs for thrombocytopenia, especially that induced by radiotherapy and chemotherapy.

\section{Abbreviations}

TMEA:

DMAG:

2, 3, 8-trimethylellagic

3, 8-dimethoxyellagic acid-2-

oxyglucoside

DOMA: $\quad$ 3, 7-Di-O-methylducheside A

TMEA-DMAG: $\quad$ 2, 3, 8-trimethylellagic-3, 8-

dimethoxyellagic acid-2-oxyglucoside
Sanguisorba

officinalis L.:

IS:

UHPLC-MS/MS: Ultra-high-performance liquid

chromatography-tandem mass

spectrometry

$\mathrm{AUC}_{(0-t)}: \quad$ Area under the plasma concentrationtime curve from time 0 to last time of quantifiable concentration

$\operatorname{AUC}_{(0-\infty)}$ : $\quad$ Area under the plasma concentrationtime curve from time 0 extrapolated to infinite time

$C_{\text {max }}$ :

$T_{\max }$ :

CL/F:

MRT:

$V_{z} / F$ :

$\mathrm{T}_{1 / 2}$ :

RSD:

LLOQ:

QC:

MRM:

FDA:

NG:

MG:

TG:

TMG:

TOG:

CYP450:

Mao:

UGT:

GST:

BCRP:

MRP2:

P-gp:

SCF/C-KIT:

$\mathrm{TPO} / \mathrm{c}-\mathrm{Mpl}$ :

JAK/STAT:

PI3K/Akt:

MEK/ERK:

GATA-1:

GATA-2:

RUNX-1:

NF-E2:

FOG-1:

FLI-1:

LncRNA:

H19:

CASC2:

AS-RBM15:

CircRNA:

MAN1A2:
Maximum concentration

The time of maximum concentration

Apparent clearance

Mean residence time

Apparent volume of distribution

Terminal half-life

Relative standard deviation

Lower limit of quantification

Quality control

Multiple reaction monitoring

Food and Drug Administration

Normal group

Model group

TMEA group

TMEA-DMAG group

TMEA-DOMA group

Cytochrome P450

Monoamine oxidase

Glucuronidase

Glutathione transferase

Breast cancer resistance protein

Multidrug resistance-associated

protein 2

P-Glycoprotein

Stem cell factor/kit ligand

Thrombopoietin/thrombopoietin receptor

Janus kinase/signal transducers and activators of transcription

Phosphatidylinositol 3 kinase/protein kinase B

Mitogen-activated protein kinase/ extracellular regulated protein kinases GATA binding protein-1

GATA binding protein-2

Runt-related transcription factor-1

Nuclear factor erythroid-2

Friend of GATA-1

Friend leukemia virus integration-1

Long non-coding RNA

Imprinted maternally expressed

transcript

Cancer susceptibility candidate 2

Antisense RNA binding protein

Circular RNA

Mannosidase alpha class $1 \mathrm{~A}$ member 2 
Plt-circR4: $\quad$ Platelet circRNA 4

MiRNA: MicroRNA.

\section{Data Availability}

The data that support the findings of this study are available from the corresponding author upon reasonable request.

\section{Conflicts of Interest}

The authors have declared no conflicts of interest.

\section{Authors' Contributions}

Wang and Wu contributed equally to this paper.

\section{Acknowledgments}

The study was supported by the National Natural Science Foundation of China (Grant nos. 81774013, 81804221, and 82074129), Science and Technology Programs of Southwest Medical University (Grant No. 2018-ZRZD-006 and 2019ZZD006), the National Major Science and Technology Project of China (Grant nos. 2018ZX09721004-006-004), the Science and Technology Planning Project of Sichuan Province, China (Grant nos. 2019JDPT0010, 2019YJ0473, 2019YJ0484, and 19PTDJ0026), Educational Commission of Sichuan Province, China (Grant nos. 18TD0051 and 18ZA0525), Science and Technology Program of Luzhou, China (Grant nos. 2018LZXNYD-ZK31, 2018LZXNYDZK49, 2019LZXNYDJ11, 2020LZXNYDP01, and 2020LZXNYDZ03), the School-Level Fund of Southwest Medical University (Grant Nos. 2017-ZRZD-017, 2017ZRQN-081, and 2018-JYJ-34).

\section{References}

[1] W. Zhou, J. Wu, Y. Zhu et al., "Study on the mechanisms of compound Kushen injection for the treatment of gastric cancer based on network pharmacology," BMC Complementary Medicine and Therapies, vol. 20, no. 1, p. 6, 2020.

[2] S. M. S. Pezeshki, N. Saki, M. V. Ghandali, A. Ekrami, and A. Y. Avarvand, "Effect of helicobacter pylori eradication on patients with ITP: a meta-analysis of studies conducted in the Middle East," Blood Research, vol. 56, no. 1, pp. 38-43, 2021.

[3] M. Zhu, X. Rong, M. Li, and S. Wang, "IL-18 and IL-35 in the serum of patients with sepsis thrombocytopenia and the clinical significance," Experimental and therapeutic medicine, vol. 19, pp. 1251-1258, 2020.

[4] K. Manasa, R. Soumya, and R. Vani, "Phytochemicals as potential therapeutics for thrombocytopenia," Journal of Thrombosis and Thrombolysis, vol. 41, no. 3, pp. 436-440, 2016.

[5] S. Zhu and Z. Chen, "General situation of treatment for primary immune thrombocytopenia with Chinese medicine and integrated Chinese and western medicine," Journal of New Chinese Medicine, vol. 52, pp. 11-14, 2020.

[6] Y. Xiong, J. Zou, and M. Yang, "Protective effect of tannins from Sanguisorba officinalis on cyclophosphamide induced myelosuppression in mice," National Product Research and Development, vol. 26, pp. 499-503, 2014.
[7] Y. Dai, X. Gao, J. Wu, X. Li, F. Huang, and W. Zou, "Effect of total saponins from Sanguisorba officinalis on megakaryocyte progenitor cells proliferation, differentiation and relative receptor expression," China Journal of Chinese Materia Medica, vol. 39, pp. 1685-1689, 2014.

[8] X. Gao, J. Wu, W. Zou, and Y. Dai, "Two ellagic acids isolated from roots of Sanguisorba officinalis L. promote hematopoietic progenitor cell proliferation and megakaryocyte differentiation," Molecules, vol. 19, no. 4, pp. 5448-5458, 2014.

[9] X. Ma, X. Guo, Y. Song et al., "An integrated strategy for global qualitative and quantitative profiling of traditional Chinese medicine formulas: baoyuan decoction as a case," Scientific Reports, vol. 6, no. 1, Article ID 38379, 2016.

[10] J. Peng, F. Tang, R. Zhou et al., "New techniques of on-line biological sample processing and their application in the field of biopharmaceutical analysis," Acta Pharmaceutica Sinica B, vol. 6, no. 6, pp. 540-551, 2016.

[11] H.-C. Jeong, J.-E. Park, J.-Y. Hyun, M.-K. Park, D.-S. Shin, and K.-H. Shin, "Determination of donepezil in human plasma using ultra performance liquid chromatographytandem mass spectrometry," Translational and Clinical Pharmacology, vol. 26, no. 2, pp. 64-72, 2018.

[12] Z. Deng, Q. Liu, W. Wu, and H. Wang, "Validation and application of a novel UHPLC-MS/MS method for the measurement of furanodienone in rat plasma," Biomedical Chromatography, vol. 34, Article ID e4717, 2020.

[13] Y. Huang, H. Lin, Y. Chen, and X. Huang, "Pharmacokinetic and bioavailability study of kurarinone in dog plasma by UHPLC-MS/MS," Biomedical Chromatography, vol. 34, Article ID e4945, 2020.

[14] Y. Lou, H. Wu, J. Zheng et al., "Determination and pharmacokinetic study of skimmin by UHPLC-MS/MS in rat plasma," Journal of Pharmaceutical and Biomedical Analysis, vol. 179, Article ID 112969, 2020.

[15] National Academy Press, Guide for the Care and Use of Laboratory Animals, National Academy Press, Washington, D.C., Institute of Laboratory Animal Resources, Commission on Life Sciences, National Research Council, 8th edition, 2011.

[16] F. Khazaei, E. Ghanbari, and M. Khazaei, "Protective effect of royal jelly against cyclophosphamide-induced thrombocytopenia and spleen and bone marrow damages in rats," Cell journal, vol. 22, no. 3, pp. 302-309, 2020.

[17] Center for Drug Evaluation and Research (CDER), Guidance for Industry on Bioanalytical Method Validation, Center for Drug Evaluation and Research (CDER), Rockville, MD, USA, 2018.

[18] Q. Zhao, Y. Zhang, G. Dai et al., "Comparison of in vivo pharmacokinetics of five constituents in Zhishi Zhizi Chi Decoction in normal rats and CUMS depressed rats," China Journal of Chinese Materia Medica, vol. 46, pp. 3687-3693, 2021.

[19] H. Liu, H. Chen, X. Qin et al., "Simultaneous determination of 5 active components of Periploca forrestii Schltr extract in rat plasma by UPLC-MS and its application to pharmacokinetic studies in normal and adjuvant-induced arthritis model rats," National Product Communications, vol. 15, pp. 1-10, 2020.

[20] Y. Jin, L. Yu, F. Xu et al., "Pharmacokinetics of active ingredients of Salvia miltiorrhiza and Carthamus tinctorius in compatibility in normal and cerebral ischemia rats: a comparative study," European Journal of Drug Metabolism and Pharmacokinetics, vol. 45, no. 2, pp. 273-284, 2020.

[21] L. Fan, X. Wang, J. Huang et al., "Comparison of the pharmacokinetic profiles of 13 phenolic acids and 6 triterpenes in normal and leukopenia rats after oral administration of 
Sanguisorba officinalis L. extract by LC-MS/MS," Journal of Separation Science, vol. 43, no. 22, pp. 4103-4122, 2020.

[22] K. Taguchi, H. Ujihira, H. Watanabe et al., "Pharmacokinetic study of adenosine diphosphate-encapsulated liposomes coated with fibrinogen $\gamma$-chain dodecapeptide as a synthetic platelet substitute in an anticancer drug-induced thrombocytopenia rat model," Journal of Pharmaceutical Sciences, vol. 102, no. 10, pp. 3852-3859, 2013.

[23] C. Liu, L. Cheng, L. Ji et al., "Intestinal microbiota dysbiosis play a role in pathogenesis of patients with primary immune thrombocytopenia," Thrombosis Research, vol. 190, pp. 11-19, 2020.

[24] R. Yin, H.-C. Kuo, R. Hudlikar et al., "Gut microbiota, dietary phytochemicals, and benefits to human health," Current Pharmacology Reports, vol. 5, no. 5, pp. 332-344, 2019.

[25] Y.-F. Li, M. Wang, X.-Y. Wang et al., "Pharmacokinetic properties of albiflorin and paeoniflorin after oral administration of pure compound, radixPaeoniae albaextract and Danggui-Shaoyao-San extract to rats," Journal of Asian Natural Products Research, vol. 13, no. 2, pp. 117-127, 2011.

[26] Y. Liu, D. Wang, G. Yang, Q. Shi, and F. Feng, "Comparative pharmacokinetics and brain distribution of magnolol and honokiol after oral administration of Magnolia officinaliscortex extract and its compatibility with other herbal medicines in Zhi-Zi-Hou-Po Decoction to rats," Biomedical Chromatography, vol. 30, no. 3, pp. 369-375, 2016.

[27] Y. Xiong and M. Yang, "Effects of sanguisorba tannins and saponins compatibility on pharmacokinetic parameters of catechin, epicatechin and ziyuglycoside I in rats," China Journal of Chinese Materia Medica, vol. 41, pp. 3661-3667, 2016.

[28] H. Zeng, P. Xue, S. Su et al., "Comparative pharmacokinetics of three major bioactive components in rats after oral administration of Typhae Pollen-Trogopterus Feces drug pair before and after compatibility," DARU Journal of Pharmaceutical Sciences, vol. 24, no. 1, p. 2, 2016.

[29] G. Liu, Q. Li, Y. Tao, and B. Zhou, "Effects of tannins extracted from fupenzi (rubus chingii hu) on activity of hepatic micosomal enzymes in rats," Chinese Architecture of Traditional Chinese Medicine, vol. 39, pp. 8-10, 2021.

[30] M. Kurata, M. Suzuki, and K. Takeda, "Effects of phenol compounds, glutathione analogues and a diuretic drug on glutathione, glutathione reductase and glutathione peroxidase from canine erythrocytes," Comparative Biochemistry and Physiology B, vol. 103, no. 4, pp. 863-867, 1992.

[31] X. Mao, L. Wu, H. Zhao et al., "Transport of corilagin, gallic acid, and ellagic acid from Fructus Phyllanthi tannin fraction in caco-2 cell monolayers," Evidence-Based Complementary and Alternative Medicine, vol. 2016, Article ID 9205379, 10 pages, 2016.

[32] K. E. Elagib, A. T. Brock, and A. N. Goldfarb, "Megakaryocyte ontogeny: clinical and molecular significance," Experimental Hematology, vol. 61, pp. 1-9, 2018.

[33] C. Gutmann, A. Joshi, A. Zampetaki, and M. Mayr, "The landscape of coding and noncoding RNAS in platelets," Antioxidants and Redox Signaling, vol. 34, no. 15, pp. 1200-1216, 2021. 